i

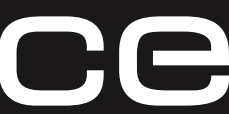

Institution of Civil Engineers

\title{
Smart surfaces by initiated chemical vapor deposition
}

Anna Maria Coclite $\mathrm{PhD}$ *

Postdoctoral Associate, Department of Chemical Engineering, Massachusetts Institute of Technology, Cambridge, MA, USA

Assistant Professor, Institute of Solid State Physics, Graz University of

Technology, Graz, Austria

The ability to modify the surface of materials with functional and responsive coatings is a powerful tool for the fabrication of smart devices for biotechnology, microfluidics, membrane technology, sensors and drug delivery systems. A recently developed method for the deposition of polymeric thin films, called initiated chemical vapor deposition (iCVD) is reviewed here. The authors will describe the high versatility of iCVD in driving application-specific properties into the material, creating a platform for the implementation of polymeric coatings into device fabrication. The significant impact of this polymerization technique lies in the possibility of obtaining polymers with chemical structure similar to the one of the polymers synthesized by conventional techniques with the advantages of a solvent-free deposition, which is totally substrate independent. Deposition has been demonstrated on paper, metal, plastics, porous substrates and very recently liquids. Tuning the process parameters allows to obtain controlled and uniform thickness over 3D substrates. Future outlook and iCVD scale-up approaches are also discussed.

\section{Introduction}

Polymer coatings offer innovative solutions for the development of functional materials for technological applications. Coating the top surface of materials with a thin film of polymer allows us to modify the interaction of the material with the environment without changing the bulk properties of the material itself, and thus increases the number of properties that a material can have. A significant research effort is devoted to the production of "smart" coatings, which dynamically respond to the stimuli coming from the environment. ${ }^{1}$ Successful examples of smart polymer surfaces are $\mathrm{pH}$ - or temperature-responsive coatings, antistain and self cleaning coatings, biomedical coatings and heterogeneous surfaces.

Wet methods have been widely used to achieve these goals. The development of dry polymerizations in which the monomers are vaporized and then polymerize on the surface (also called chemical vapor deposition [CVD]) aims to complement and enlarge the applicability of polymer coatings to the fields where the presence of solvent is detrimental. ${ }^{2}$ When the polymerization takes place from the vapor phase, the tridimensional features of the substrate can be uniformly coated and reproduced without the typical side effects of the presence of liquid solvents such as meniscus formation, bridging or pooling. The thermal issues associated with drying or curing are avoided and many of the vapor-phase polymerizations happen at low substrate temperature, allowing the coating of thermo-labile substrates. Adjusting the system parameters, the polymers obtained by vapor phase can have tailored chemical and physical properties, precise control over thickness and conformality. ${ }^{3}$

The reaction mechanism of the dry polymerizations is similar to the wet ones. In the free-radical chain-growth polymerization, the first step is the initiation step in which active species (e.g. ions, radicals, 
fragments) are created to react with the monomer creating active sites on the monomer molecules. The second step is the propagation: the active monomer molecules interact with other monomer molecules and the polymeric chain starts growing. Differently from liquid-phase polymerization, the growing polymeric chains are adsorbed on a cooled substrate. The third step is the termination, where the radical end of the growing polymer chain is capped by reaction with another radical. The CVD free-radical polymerization techniques differ in the initiation step. In plasma-enhanced $\mathrm{CVD}^{4}$ and in hot-wire CVD, ${ }^{5}$ the radicals are created by direct fragmentation of the monomer molecules by the energetic electrons of the plasma discharge or by high temperatures, respectively. Direct fragmentation of the monomer can result in the cleavage of the monomer functional groups negatively affecting polymer properties.

In the past 10 years, a new CVD method has been developed, termed initiated CVD (iCVD). ${ }^{3,6-8}$ In the iCVD process, the radicals are not created by fragmentation of the monomer molecules but of an initiator. An initiator is a chemical compound that has a labile bond (e.g. the O-O bond in peroxides), which undergoes thermal decomposition at a relatively low temperature. The monomer and the initiator are fed to a reactor chamber in which a filament array is suspended $\sim 2 \mathrm{~cm}$ above a substrate holder-heat-exchange plate. The filament is heated to the initiator decomposition temperature, which is generally below $300^{\circ} \mathrm{C}$. In comparison, the decomposition temperature of the most monomers are above $400^{\circ} \mathrm{C}$. The low-energy input required to fragment the initiator ensures that the monomer molecules remain intact and fully retain all the functionalities. The radicals of the initiator react with the vinyl bond of the monomer molecules adsorbed on the substrate surface to initiate the polymerization, therefore the chain-growth step takes place on the surface, as shown in Scheme 1.

In numerous articles, it has been demonstrated that iCVD is substrate-independent and takes place at low substrate temperature (between $15-60^{\circ} \mathrm{C}$ ). Polymers have been successfully deposited on paper, ${ }^{9,10}$ plastic, ${ }^{11,12}$ liquids ${ }^{13-16}$ (see Figure 1a), poly-dimethylsiloxane (PDMS), ${ }^{17,18}$ gold nanorod. ${ }^{19}$ The resulting polymers have full functionality retention and can be obtained at high-deposition rates. The absence of catalysts, plasticizers, solvents and dispersants improves the biocompatibility of iCVD polymers, which

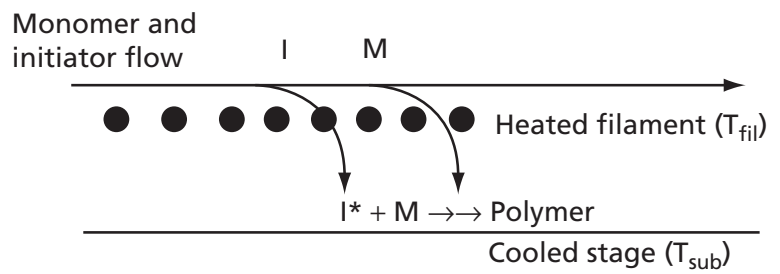

Scheme 1. Schematics of the iCVD system. iCVD, initiated chemical vapor deposition. I, initiator; $M$, monomer; $T_{\text {fil }}$, filament temperature; $\mathrm{T}_{\text {sub' }}$ substrate temperature. makes this process very attractive for biomedical applications. ${ }^{20,21}$ The deposition parameters can be modified in order to obtain different coating profiles on tridimensional substrates, depending on the application. Figure $1 \mathrm{~b}$ shows a planarizing polymer that can be used as pore filler, ${ }^{22}$ and to smooth the asperities of rough substrates..$^{23}$ In this case, the coating was thicker in the pores or valleys of the substrate and thinner on the higher features. On the contrary, Figure 1c shows that it is possible to obtain also very high conformality: in this case, the polymer had a uniform thickness on the top, side and on the bottom of the trenches. ${ }^{24}$
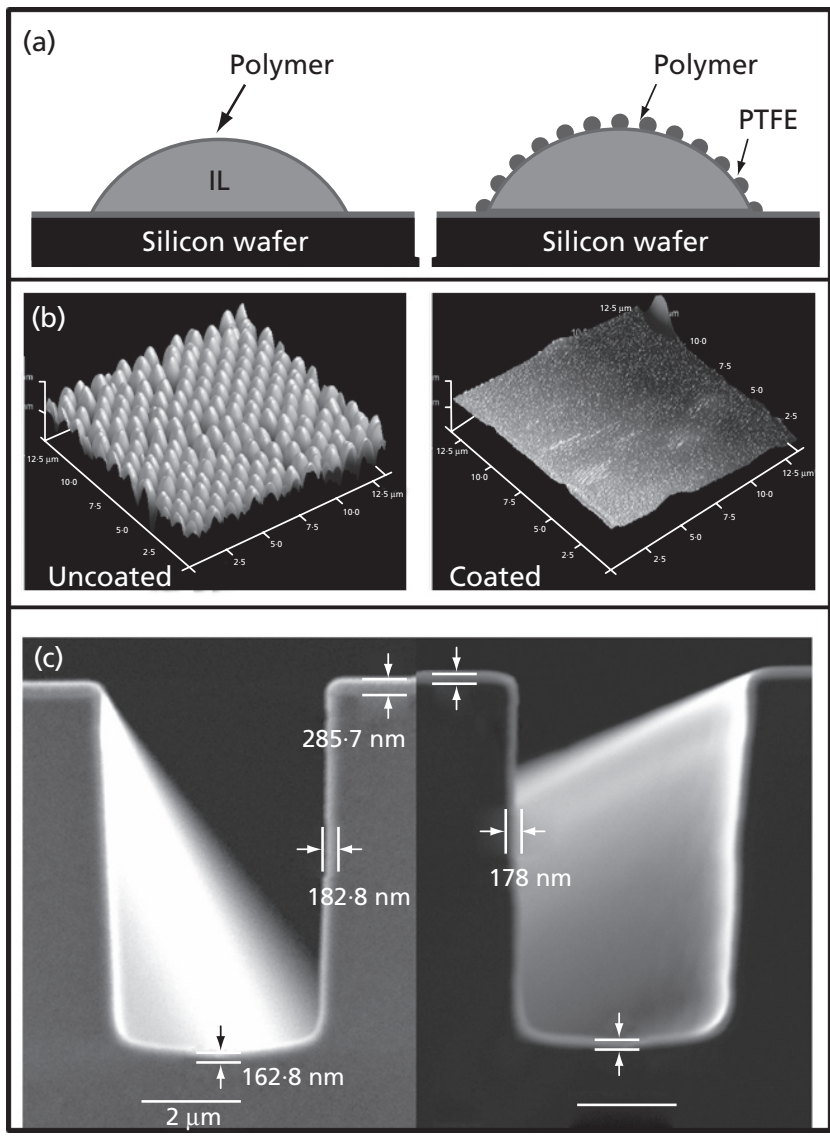

Figure 1. (a) Deposition of iCVD polymer on the surface of IL droplet without and with PTFE particles. The PFTE particles facilitate polymerization onto the entire surface area of the IL. Adapted with permission from Ref. 13. Copyright (2012) American Chemical Society. (b) Deposition of a organosilicon polymer coating on top of microspheres with diameter $\sim 1 \mu \mathrm{m}$. The polymer completely filled all the gaps between the spheres giving a microscopically flat surface. Adapted with permission from Ref. 23. Copyright (2012) American Institute of Physics. (c) Deposition of a conformal polymer coating inside the trenches of a silicon wafer. The coating is uniformly distributed on top, wall and bottom of the trenches. Adapted with permission from Ref. 24. Copyright (2012) American Chemical Society. iCVD, initiated chemical vapor deposition; IL, ionic liquid; PTFE, poly(tetrafluoroethylene). 
Smart surfaces have been obtained by iCVD ranging from super hydrophobic coating, ${ }^{25-27}$ antifouling ${ }^{28,29}$ and stimuli-responsive polymers for drug delivery ${ }^{30}$ or protein adsorption. ${ }^{31}$ Many polymers have been deposited by iCVD, for example, temperature-sensitive poly ( $N$-isopropylacrylamide) (p(NIPAAm)), ${ }^{32-34} \mathrm{pH}$-sensitive methacrylic acid (MAA) copolymers ${ }^{35}$ or poly(hydroxyethyl methacrylate) (p(HEMA)) hydrogels. ${ }^{36,37}$ Here, some specific applications of iCVD smart polymers will be reviewed, emphasizing the aspects that are difficult to obtain with other polymerization techniques.

\section{Functional coating}

The term "functional" in this context refers to that class of coating characterized by some native properties and functions that make them of high technological interest. The result of combining a functional coating and a substrate, which has some other complementary properties, is a multifunctional, or smart, material. iCVD has been demonstrated to be very successful for the development of multifunctional materials. For example, it was useful in adding super-hydrophobicity and oleophobicity to stretching fabric, ${ }^{38}$ antifouling properties to membranes ${ }^{31}$ or barrier properties to plastic. ${ }^{39}$

\subsection{Superhydrophobic coatings}

Superhydrophobic coatings have been obtained by iCVD, as polymers of $1 \mathrm{H}, 1 \mathrm{H}, 2 \mathrm{H}, 2 \mathrm{H}$-perfluorodecyl acrylate (PFDA). ${ }^{15,25,26}$ This monomer contains a long fluorinated chain, $-\left(\mathrm{CF}_{2}\right)_{7} \mathrm{CF}_{3}$, that is totally preserved during the iCVD process. The long fluorinated chains pending from the polymer backbone have a natural tendency to reorient toward themselves and form a crystalline bilayer structure, called smectic B phase. By tuning the iCVD parameters (filament temperature, initiator over monomer ratio and substrate temperature), it is possible to tune also the degree of crystallinity in the film. ${ }^{26}$ Semicrystalline polymers give stable surface properties, without hydrophobic recovery and create generally rough surfaces (root mean square roughness higher than $30 \mathrm{~nm}$ ), which can be useful for enhancing cell growth or enhancing the hydrophobicity of the surface. It has been demonstrated that grafting the polymeric $\mathrm{p}(\mathrm{PFDA})$ chains to the surface, that is, covalently bonding the chains to the substrate, produces oleophobic $\mathrm{p}$ (PFDA) surfaces. ${ }^{25}$ Figure $2 \mathrm{a}$ shows the cosine of the contact angle (CA) when the surface was wetted with several hydrocarbon oils with different liquid-surface energies. The grafted polymer exhibits higher CAs than the ungrafted one. p(PFDA) superhydrophobic properties were used to coat a paper solar cell so that the device could produce power under light irradiation even when totally submerged in water. ${ }^{9}$ Free-standing $\mathrm{p}$ (PFDA) polymer films have been obtained by depositing the iCVD polymer over droplets of ionic liquid. ${ }^{15}$ Ionic liquids are non volatile solvents, hence compatible with vacuum systems. Other semicrystalline structures have been obtained by soft-epitaxy: depositing poly(tetrafluoroethylene) by iCVD over the surface of carbon nanotube. In this case, modifying the substrate topography, it was possible to modify the crystal structure of the polymer. ${ }^{40}$

\subsection{Coatings for microfluidic devices}

p(PFDA) coatings have also been applied on PDMS-based microfluidic devices in order to create a barrier coating and increase the chemical compatibility of the PDMS, preventing the lateral diffusion of the chemicals. ${ }^{41}$ This type of iCVD-modified microfluidic devices have been demonstrated to be successful for synthesis, and reactor miniaturization. ${ }^{16}$ The microchannels have the potential to provide superior control over mixing as compared with traditional batch reactions. The high conformality of iCVD coatings makes the integration of iCVD polymers on microfluidic devices a powerful tool to modify the surface of the microchannels without occluding them. A polymer of maleic anhydride was deposited on microfluidic lung-assisted device, and it allows for the selective permeation of oxygen and carbon dioxide while acting as a liquid barrier to prevent the permeation of blood of the vascular vessels (see Figure 2b). ${ }^{18,42}$ iCVD polymers were also used to bond some microchannels together: one side of the device was coated with poly(glycidyl methacrylate) $\mathrm{p}(\mathrm{GMA})$ and the other side with poly(4-aminostyrene) $\mathrm{p}(\mathrm{AS}) .^{43}$ The epoxy groups of $\mathrm{p}(\mathrm{GMA})$ were reacted with the amino groups of the $\mathrm{p}(\mathrm{AS})$ to assemble the device. Microchannels were also produced by using the UV-responsive properties of the poly(o-nitrobenzyl methacrylate) (PoNBMA) deposited by iCVD. ${ }^{44}$ PoNBMA is hydrophobic but nitrobenzyl groups can be cleaved to form patterned surfaces with hydrophilic channels. Porous chromatography paper was coated with PoNBMA and irradiated with UV light to create hydrophilic channels surrounded by hydrophobic walls. The ability to deposit on porous substrates and coat the internal pores was used to sequentially deposit functional polymer coatings onto paper-based microfluidic devices, retaining the fibrous morphology necessary to generate capillary-driven flow. ${ }^{10}$ A hydrophilic polymer coating with a high surface concentration of ionizable groups was deposited onto the surface of the paper fibers in order to passively separate analytes, into its anionic and cationic components. Sequentially, the UV-responsive PoNBMA was deposited to act as a responsive hydrophobic/hydrophilic switch to block or allow the path of fluid within the devices.

\subsection{Antifouling coatings}

Figure $2 \mathrm{c}$ shows the antifouling properties of the poly(ethylene oxide) (PEO) deposited by iCVD. The nonspecific protein adsorption was much smaller on the iCVD PEO surface than on other hydrophilic surfaces tested (i.e. silicon, amine- and hydroxyl-terminated surfaces). This was attributed to the greater wettability and hydrophilicity of the grafted iCVD PEO surfaces that were effective in minimizing the protein adsorption. Good antifouling properties were also achieved with zwitterionic coatings deposited by iCVD. ${ }^{29}$ These coatings were used to prevent the fouling of reverse osmosis membranes for water desalination. 


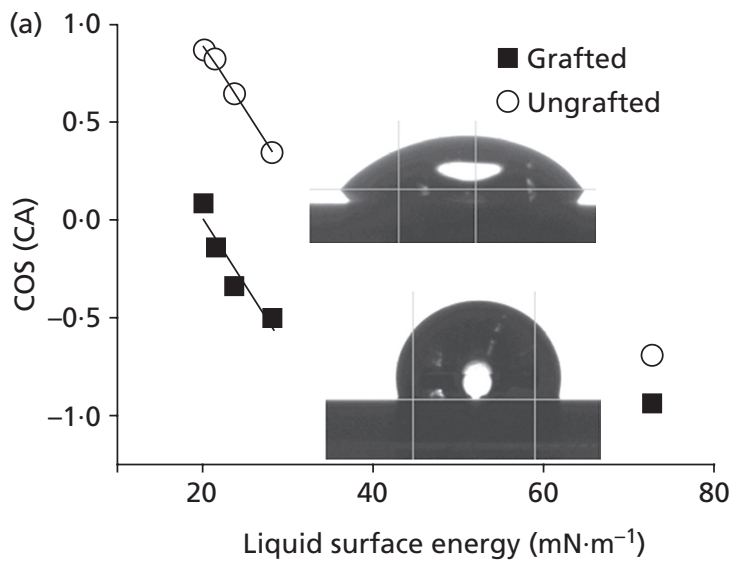

(b)

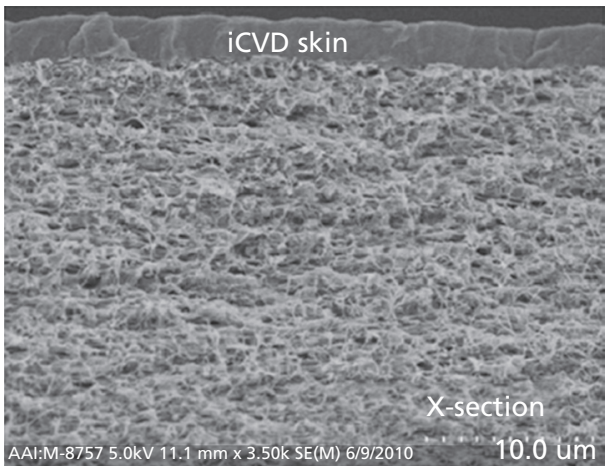

Liquid surface energy $\left(\mathrm{mN} \cdot \mathrm{m}^{-1}\right)$

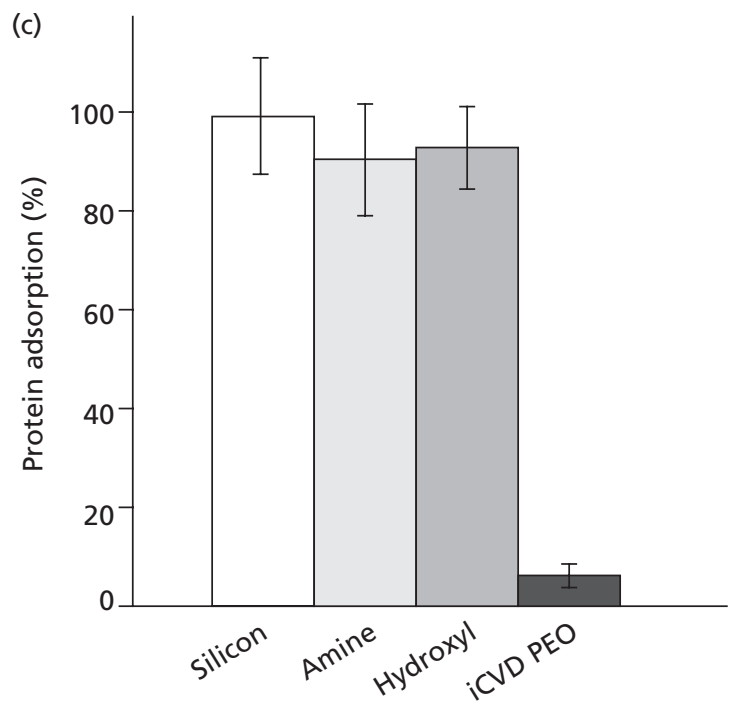

Figure 2. (a) Plot of the cosine of the CA of the grafted and ungrafted $p$ (PFDA) as a function of the liquid surface energy of the hydrocarbon oils used to wet the surfaces. The plot shows that the grafted $p$ (PFDA) surface has better oleophobic properties than the ungrafted one. Adapted with permission from Ref. 25. Copyright (2012) Wiley. (b) iCVD skin deposited on top of PTFE membrane to have selective permeation of gas (i.e. $\mathrm{O}_{2}$ and $\mathrm{CO}_{2}$ ) and act as liquid barrier. Adapted with permission from Ref. 42. Copyright (2012) Elsevier. (c) Histogram of the protein adsorption percentage of different hydrophilic surfaces: silicon wafer, amine and hydroxyl-terminated surfaces and iCVD-deposited PEO. The better antifouling properties of iCVD PEO is due to its enhanced wettability and hydrophilicity. Adapted with permissions from 28. Copyright (2012) Wiley. CA, contact angles; iCVD, initiated chemical vapor deposition; PTFE, poly(tetrafluoroethylene); poly(ethylene oxide) (PEO).

\subsection{Permeation barrier coating}

An iCVD barrier coating made of $\mathrm{p}(\mathrm{GMA})$ was deposited on $\mathrm{NaCl}$ particles with metallic coatings to decrease the dissolution rate of the bare $\mathrm{NaCl}^{39}$ The ability to coat particles is a particular strength of $\mathrm{iCVD}$, since it is a non line-of-sight deposition that allows uniform coating of particles. This prevents the agglomeration that is often observed by wet methods. The combination of iCVD with the richness of organic chemistry allowed the design of an impermeable and hard but flexible coating. This was obtained as a result of copolymerization of $\mathrm{p}(\mathrm{MA})$ and $\mathrm{p}(\mathrm{AS})$, followed by annealing. 
The annealing resulted in cross-linking of the polymeric chains by reaction of the anhydride and the amino groups contained in the copolymer.

iCVD was also demonstrated to be easily integrated in multilayer deposition technology. The dryness of this process is beneficial to avoid contamination and/or dissolution of the underlayers in multistack structures. iCVD polymers were coupled with inorganic layer deposition to obtain multilayer barrier structures on plastics and achieve the stringent barrier requirements of flexible electronics. ${ }^{45}$ iCVD polymers were demonstrated to be resistant to the subsequent deposition of inorganic layers ${ }^{46}$ so that they could be sandwiched between two inorganic layers and fill the pores of the bottom layer. ${ }^{47}$ iCVD organosilicon polymers were also demonstrated to give global and local planarization (as shown in Figure 1b), which is useful to make the surface of the plastic substrate totally flat for subsequent deposition of the successive inorganic layers..$^{23,48}$

\subsection{Electronic applications of iCVD polymer}

iCVD polymers have been successfully applied to electronic applications, as insulating dielectrics as well as conducting polymers. Poly(meta-diethynyl benzene) was deposited by iCVD with high molecular weight. This material has dielectric constant $(\mathrm{k})$ between 2.3 and 3.2 in the visible wavelength range, which classifies it as a "low-k" dielectric polymer. Low dielectric constants were also obtained by creating air gaps after the decomposition of the iCVD polymer to form voids. ${ }^{49}$

iCVD poly(2-hydroxyethyl methacrylate) (PHEMA) was used as a polymer electrolyte to substitute the liquid electrolyte in dyesynthesized solar cells. ${ }^{51}$ The successful integration of the iCVD PHEMA was due to its pore filling capabilities, so it could fill in the pores of the titanium dioxide used in the solar cells. ${ }^{22}$ The cellincorporating $\mathrm{TiO}_{2}$ filled with the iCVD polymer showed better performance, compared with the cell filled with liquid electrolyte that is attributed to a lower rate of electron recombination.

Finally, Bakker et al. ${ }^{51}$ demonstrated that by doping the iCVDdeposited oligo(phenyl acetylene) with iodine vapors, it is possible to obtain conductive polymers. These polymers exhibit electronic conductivities comparable with the values already reported in literature for solution-processed polymers. Proton conductivity similar to the one of the commercial available Nafion membranes was obtained through a membrane made of a copolymer of MAA and PFDA deposited by iCVD. ${ }^{11}$ The PFDA units were responsible of creating a hydrophobic backbone to ensure enough membrane stability, while the acid units with ionizable groups created the ionic channels for the proton passage.

\section{Responsive coatings}

Responsive coatings are characterized by dynamic switching of their properties depending on external stimuli (e.g. electrical field, temperature, $\mathrm{pH}$ ). Nature is full of example of responsive materials, such as the skin of lizard or cuttlefish, which change their body patterns and colors depending on the surrounding environment. ${ }^{53}$ Recently, numerous synthetic examples of responsive surfaces have emerged, which rely on conformational change in the polymer network, or pattern change. The retention of the functional groups

(a)

$$
\frac{T<\text { LCST }}{\text { Diffusion }} \quad \frac{T>\text { LCST }}{\text { Adsorption }}
$$

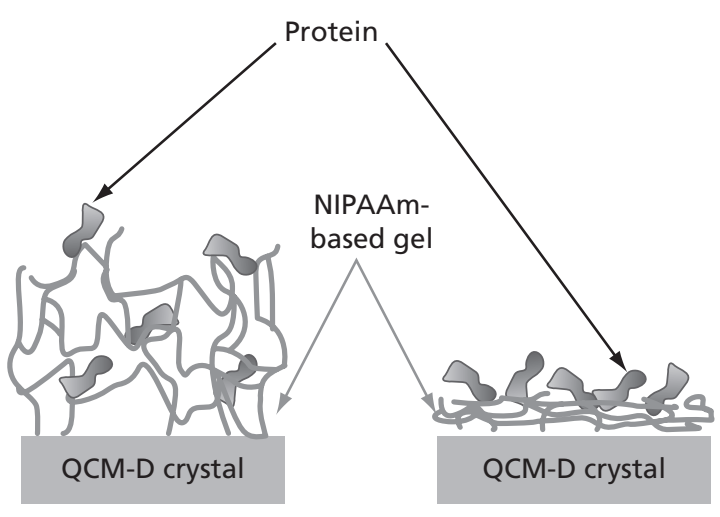

(b)

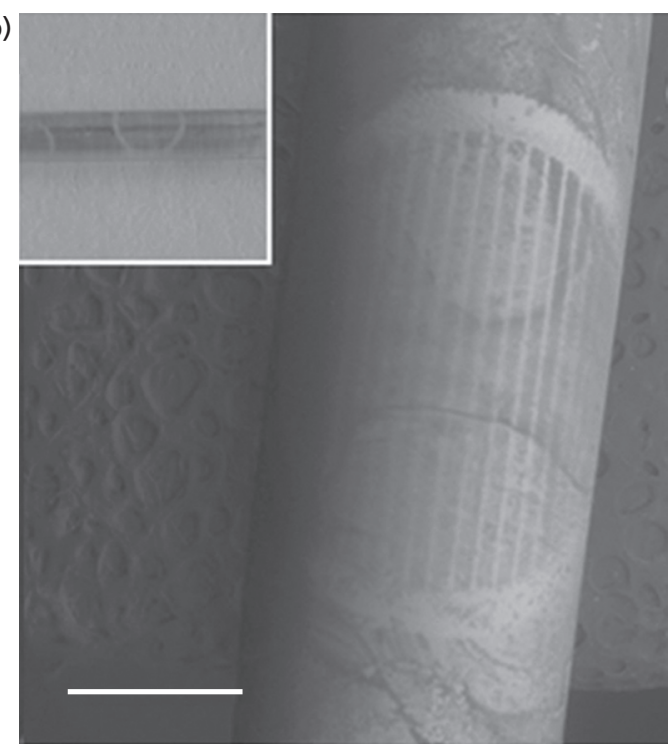

Figure 3. (a) QCM-D was used to investigate the thermo-responsive properties of p(NIPAAm). Below the LCST the polymer is in its expanded coil configuration where the proteins diffuse into the network. Above the LCST the polymer is in its collapsed state in which only surface adsorption is observed. Adapted from Ref. 32. Copyright (2012) American Chemical Society. (b) Patterning of a curved metal substrate using lithography of iCVD polymer. Adapted from Ref. 63. Copyright (2012) Wiley. LCST, lower critical solution temperature; p(NIPAAm), poly( $N$-isopropylacrylamide); QCM-D, quartz crystal microbalance with dissipation monitoring. 
of polymers is critical to achieve the desired response, and this is the reason why iCVD plays an important role for the synthesis of this class of material.

A polymer known for its thermo-responsive properties is the p(NIPAAm). This polymer is characterized by a lower critical solution temperature (LCST) about $32^{\circ} \mathrm{C}$, which is similar to body temperature and therefore makes the responsive properties of this polymer of high biomedical interests. Below the LCST the polymer is in a swollen state, also called extended coil configuration, in which the amide groups are exposed to the surface, resulting in high hydrophilicity. Above the LCST, the polymer is in a globular configuration in which the inter- and intra-chain amide groups are bonded, and the hydrophobic isopropyl groups are exposed to the surface. p(NIPAAm) deposited by iCVD gave $30^{\circ}$ water CA (WCA) difference from the hydrophilic to the hydrophobic state when deposited on flat silicon surfaces and $85^{\circ}$ WCA difference when the polymer was deposited on carbon nanotube forest. ${ }^{32,34}$ Alf et al. demonstrated that when the p(NIPAAm) deposited by iCVD is in its swollen state (i.e. below the LCST) the proteins are not adsorbed on the surface but diffuse into the polymer network, as shown in Figure 3a. Above the LCST, when the polymer is in its globular configuration, the proteins show simple monolayer adsorption. This property was used to design microgrooves for tissue construct formation. ${ }^{54}$ The $\mathrm{p}$ (NiPAAm) was deposited into the microgrooves of a PDMS substrate. The hydrophilic and swollen state of the polymer was used to seed the cells. Tissue formation occurred above the LCST, when the polymer was in its hydrophobic and collapsed state. The tissue constructs were then retrieved bringing the p(NIPPAm) back to its hydrophilic and swollen state, resulting in longitudinal tissue contacts.

\subsection{Applications as sensors}

Sensors are also based on responsive properties. A sensor for nitroaromatic explosives was developed using the dynamic swelling ability of poly(4-vinylpyridine) (P4VP).$^{55}$ The device was composed of two electrodes separated by dielectric $\mathrm{SiO}_{2}$. A polymer line is deposited between the electrodes with a longitudinal gold contact overlaying on top. In the presence of nitroaromatic compounds, the polymer swells deforming the Au line, hence change its resistance. Another example of sensors, made by iCVD, was developed by Ozaydin-Ince et al. ${ }^{56}$ In this case, the optode sensor was encapsulated in microworms of $\mathrm{p}$ (HEMA) for in vivo detection of sodium. They demonstrated that $\mathrm{p}$ (HEMA) encapsulation does not interfere with the activity of the optode sensor but allows the sensors to remain at the injection site.

\subsection{Hydrogels for biotechnology and drug delivery} $\mathrm{p}$ (HEMA) belongs to a class of polymers called hydrogels. Hydrogels are known for their dynamic swelling response to aqueous environments. This results in changes in the mechanical properties, protein adsorption capabilities and hydrophilicity of the polymer. Free-standing p(HEMA) at high molecular weight was deposited by iCVD and resulted in the prevention of nonspecific protein adsorption and cells proliferation. ${ }^{36,37}$ Free-standing p(HEMA) and p(NIPAAm) were also deposited by Frank-Finney et al. ${ }^{14}$ using ionic liquids and silicone oils as substrates. The fast and reversible changes in the thickness of p(HEMA) were used in an impedance sensor to control the passage of the analyte..$^{57} \mathrm{By}$ regulating the cross-link density of the p(HEMA) hydrogels, it is possible to systematically control the mesh size of the polymer and therefore allow the selective permeation of some analytes while preventing the passage of other species (e.g. foulant in biosensing applications). ${ }^{58}$

The responsive properties of $\mathrm{p}$ (HEMA) have been also used for controlled drug delivery systems. Coaxial nanotubes were fabricated by iCVD with a hydrogel core and a shape memory shell (SMP) for burst release of fluorescein. The nanopores of an anodic aluminum oxide (AAO) membrane were conformally coated with a SMP first and then with hydrogel. When the membrane was etched, the coaxial nanofilms were released. A fluorescent dye was encapsulated and absorbed by the hydrogel layer. At elevated temperature, the burst release of the fluorescent dye occurs due to the stress applied by the shape memory outer layer. Conformal deposition of the iCVD polymer in the pores of AAO membranes was also used to narrow down the pores of the membrane to few nm and achieve hydrophobicity-based separation of analytes. ${ }^{12}$ Controlled release of a crop protection compound in the field was obtained by encapsulating the compound microparticles in an acrylate-based iCVD layer. $^{58}$

Examples of pH-responsive polymers deposited by iCVD have been demonstrated by Karaman et al. ${ }^{35}$ and McInnes et al. ${ }^{30}$ In the first contribution, the authors demonstrated that the as-deposited poly(2diisopropylamino)ethyl methacrylate shows reversible switching of WCA values between 87 and $28^{\circ}$ after successive treatments of high- and low-pH water solutions, respectively. Higher WCA differences were obtained when the same polymer was deposited on electrospun fiber mat. In the second contribution, McInnes et al. show that the $\mathrm{pH}$-dependent properties of a copolymer of MAA and ethylene dimethacrylate (p(MAA-co-EDMA)) was used to fabricate a drug delivery system: a model drug was loaded into the pores of a silicon matrix and then capped with the copolymer. At low $\mathrm{pH}$, the release of the drug was four times slower, while at neutral $\mathrm{pH}$ the drug could be quickly delivered. The importance of such system is that it may enable site-specific targeting for drug therapy.

\subsection{Patterned coatings}

Patterned surfaces are frequently found in nature, for example on the feet of geckos. These are regular patterned surfaces that can create a switchable adhesive force, which allows the geckos to climb walls. Regular patterns were created by depositing the iCVD polymer on a surface with patterns of transition salt inhibitors. ${ }^{60}$ 
The underlying salt prevents polymerization, allowing to control the location of polymer. The combination of the patterning technique on tridimensional porous substrates may bring a new generation of hydrophobic, click-active and light-responsive polymers onto specific regions of the substrate. Another approach used to create regular patterns on the substrates consisted of stretching and releasing an elastic PDMS substrate coated with iCVD polymers having higher stiffness. ${ }^{61}$ Patterns of self-assembly of pillars were obtained by depositing a hydrophilic polymer on the surface of hydrophobic PDMS pillars. ${ }^{62}$ Submerging them in water and then letting the water evaporate induces the assembly of pillars into regular patterns. Finally, iCVD polymers were also used to create patterns on curved metal substrates, where conventional soft lithography is poorly applied (see Figure $3 \mathrm{~b}$ ). ${ }^{63} \mathrm{~A}$ photosensitive polymeric thin film was deposited directly on nonplanar substrates by iCVD and then developed to selectively remove masked regions, creating a negative-tone patterned surface.

\section{Conclusions and future outlook}

Selected applications of iCVD coatings have been reviewed. iCVD offers many different advantages, which are difficult to achieve with other deposition techniques. Pure polymers (i.e. free of leachable chemicals) with chemical structures similar to those synthesized in solution can be deposited at high-deposition rates, with controlled thickness and conformality, on every kind of substrate. This results in wide applicability of this deposition technique for thin film manufacture. Functional and responsive coatings deposited by iCVD have been applied to solve technological challenges in biotechnology, electronics, microfluidics, drug delivery, sensors and membranes.

The newly demonstrated possibility of combining iCVD polymerization over liquid "substrates" opens up a wide variety of new technological applications, that is, electrolyte membranes in fuel cells, nonvolatile electrolytes in batteries and ionic liquid core-polymer shell particles for use in optical and chemical applications.

Thanks to chemical kinetics and fluid dynamics investigations of iCVD polymer reactors, the scale-up from lab to industry scale has been successful for batch processing as well as for roll-to-roll processing on flexible substrates such as plastics, papers, textiles and membranes. For commercialization, the all-dry nature of CVD has the potential to reduce environmental, health and safety impacts associated with solvents as well as the economic costs associated with solvent disposal. All these factors make iCVD a rapidly growing technique for the deposition of polymer coatings and device fabrications.

\section{Acknowledgements}

The author would like to thank Prof. Karen Gleason and Prof. Ayse Asatekin for large scientific contributions.

\section{REFERENCES}

1. Baghdachi, J. Smart coatings. In Smart Coatings II, Vol. 1002. Washington: American Chemical Society, 2009, 3-24.

2.Seidel, S.; Riche, C. T.; Gupta, M. Chemical vapor deposition of polymer films. In Encyclopedia of Polymer Science and Technology. New Jersy: John Wiley \& Sons, Inc., 2002.

3. Ozaydin-Ince, G.; Coclite, A. M.; Gleason, K. K. CVD of polymeric thin films: applications in sensors, biotechnology, microelectronics/organic electronics, microfluidics, MEMS, composites and membranes. Reports on Progress in Physics 2012, 75 (1), 1-40.

4. Forch, R.; Chifen, A. N.; Bousquet, A.; Khor, H. L.; Jungblut, M.; Chu, L. Q.; Zhang, Z.; Osey-Mensah, I.; Sinner, E. K.; Knoll, W. Recent and expected roles of plasmapolymerized films for biomedical applications. Chemical Vapor Deposition 2007, 13(6-7), 280-294.

5. Lau, K. K. S.; Lewis, H. G. P.; Limb, S. J.; Kwan, M. C.; Gleason, K. K. Hot-wire chemical vapor deposition (HWCVD) of fluorocarbon and organosilicon thin films. Thin Solid Films 2001, 395(1-2), 288-291.

6. Yagüe, J. L.; Coclite, A. M.; Petruczok, C.; Gleason, K. K. Chemical vapor deposition for solvent-free polymerization at surfaces. Macromolecular Chemistry and Physics 2012, DOI: 10.1002/macp.201200600.

7. Alf, M. E.; Asatekin, A.; Barr, M. C.; Baxamusa, S. H.; Chelawat, H.; Ozaydin-Ince, G.; Petruczok, C. D.; Sreenivasan, R.; Tenhaeff, W. E.; Trujillo, N. J.; Vaddiraju, S.; $\mathrm{Xu}$, J. J.; Gleason, K. K. Chemical vapor deposition of conformal, functional, and responsive polymer films. Advanced Materials 2010, 22(18), 1993-2027.

8. Im, S. G.; Gleason, K. K. Solvent-free modification of surfaces with polymers: the case for initiated and oxidative chemical vapor deposition (CVD). AlChE Journal 2011, 57, 276-285.

9. Barr, M. C.; Rowehl, J. A.; Lunt, R. R.; Xu, J.; Wang, A.; Boyce, C. M.; Im, S. G.; Bulovic, V.; Gleason, K. K. Direct monolithic integration of organic photovoltaic circuits on unmodified paper. Advanced Materials 2011, 23 3500-3505.

10. Kwong, P.; Gupta, M. Vapor phase deposition of functional polymers onto paper-based microfluidic devices for advanced unit operations. Analytical Chemistry 2012, 84(22), 10129-10135.

11. Coclite, A. M.; Lund, P.; Di Mundo, R.; Palumbo, F. Novel hybrid fluoro-carboxylated copolymers deposited by initiated chemical vapor deposition as protonic membranes. Polymer 2013, 54(1), 24-30.

12. Asatekin, A.; Gleason, K. K. Polymeric nanopore membranes for hydrophobicity-based separations by conformal initiated chemical vapor deposition. Nano Letters 2011, 11, 677-686.

13. Bradley, L. C.; Gupta, M. Encapsulation of ionic liquids within polymer shells via vapor phase deposition. Langmuir $\mathbf{2 0 1 2}$, 28(27), 10276-10280. 
14.Frank-Finney, R. J.; Haller, P. D.; Gupta, M. Ultrathin free-standing polymer films deposited onto patterned ionic liquids and silicone oil. Macromolecules 2012, 45(1), 165-170.

15. Haller, P. D.; Frank-Finney, R. J.; Gupta, M. Vapor-phase free radical polymerization in the presence of an ionic liquid. Macromolecules 2011, 44(8), 2653-2659.

16. Lazarus, L. L.; Riche, C. T.; Marin, B. C.; Gupta, M.; Malmstadt, N.; Brutchey, R. L. Two-phase microfluidic droplet flows of ionic liquids for the synthesis of gold and silver nanoparticles. Acs Applied Materials \& Interfaces 2012, 4(6), 3077-3083.

17. Yin, J.; Yagüe, J. L.; Eggenspieler, D.; Gleason, K. K.; Boyce, M. C. Surface micropatterning: deterministic order in surface micro-topologies through sequential wrinkling. Advanced Materials 2012, 24(40), 5440-5440.

18. Sreenivasan, R.; Bassett, E.; Cervantes, T.; Hoganson, D.; Vacanti, J.; Gleason, K. Solvent-free surface modification by initiated chemical vapor deposition to render plasm bonding capabilities to surfaces. Microfluidics and Nanofluidics 2012, 12(5), 835-839.

19. Jo, W.; Freedman, K.; Yi, D. K.; Bose, R. K.; Lau, K. K. S.; Solomon, S. D.; Kim, M. J. Photon to thermal response of a single patterned gold nanorod cluster under near-infrared laser irradiation. Biofabrication 2011, 3(1), 015002-015009.

20. Achyuta, A. K. H.; Polikov, V. S.; White, A. J.; Lewis, H. G. P.; Murthy, S. K. Biocompatibility assessment of insulating silicone polymer coatings using an in vitro glial scar assay. Macromolecular Bioscience 2010, 10(8), 872-880.

21. Achyuta, A. K. H.; Stephens, K. D.; Lewis, H. G. P.; Murthy, S. K. Mitigation of reactive human cell adhesion on poly(dimethylsiloxane) by immobilized trypsin. Langmuir 2010, 26(6), 4160-4167.

22. Nejati, S.; Lau, K. K. S. Pore filling of nanostructured electrodes in dye sensitized solar cells by initiated chemical vapor deposition. Nano Letters 2011, 11(2), 419-423.

23. Coclite, A. M.; Gleason, K. K. Global and local planarization of surface roughness by chemical vapor deposition of organosilicon polymer for barrier applications. Journal of Applied Physics 2012, 111(7), 073516-1-073516-7.

24. Xu, J.; Gleason, K. K. Conformal polymeric thin films by lowtemperature rapid initiated chemical vapor deposition (iCVD) using tert-butyl peroxybenzoate as an initiator. ACS Applied Materials \& Interfaces 2011, 3, 2410-2416.

25. Coclite, A. M.; Shi, Y.; Gleason, K. K. Grafted crystalline poly-perfluoroacrylate structures for superhydrophobic and oleophobic functional coatings. Advanced Materials 2012, 24(33), 4534-4539.

26. Coclite, A. M.; Shi, Y. J.; Gleason, K. K. Controlling the degree of crystallinity and preferred crystallographic orientation in poly-perfluorodecylacrylate thin films by initiated chemical vapor deposition. Advanced Functional Materials 2012, 22(10), 2167-2176.
27. Takachi, M.; Yasuoka, H.; Ohdaira, K.; Shimoda, T.; Matsumura, H. A novel patterning technique using superhydrophobic PTFE thin films by Cat-CVD method. Thin Solid Films 2009, 517(12), 3622-3624.

28. Bose, R. K.; Nejati, S.; Stufflet, D. R.; Lau, K. K. S. Graft polymerization of anti-fouling PEO surfaces by liquid-free initiated chemical vapor deposition. Macromolecules 2012, 45, 6915-6922.

29. Yang, R.; Xu, J.; Ozaydin-Ince, G.; Wong, S. Y.; Gleason, K. K. Surface-tethered zwitterionic ultrathin antifouling coatings on reverse osmosis membranes by initiated chemical vapor deposition. Chemistry of Materials 2011, 23, 1263-1272.

30. McInnes, S. J. P.; Szili, E. J.; Al-Bataineh, S. A.; Xu, J.; Alf, M. E.; Gleason, K. K.; Short, R. D.; Voelcker, N. H. Combination of $\mathrm{iCVD}$ and porous silicon for the development of a controlled drug delivery system. ACS Applied Materials \& Interfaces 2012, 4(7), 3566-3574.

31. Yang, R.; Asatekin, A.; Gleason, K. K. Design of conformal, substrate-independent surface modification for controlled protein adsorption by chemical vapor deposition (CVD). Soft Matter 2012, 8(1), 31-43.

32. Alf, M. E.; Hatton, T. A.; Gleason, K. K. Novel $\mathrm{N}$-isopropylacrylamide based polymer architecture for faster LCST transition kinetics. Polymer 2011, 52, 4429-4434.

33. Alf, M. E.; Hatton, T. A.; Gleason, K. K. Insights into thin, thermally responsive polymer layers through quartz crystal microbalance with dissipation. Langmuir 2011, 27, 10691-10698.

34. Alf, M. E.; Hatton, T. A.; Gleason, K. K. Initiated chemical vapor deposition of responsive polymeric surfaces. Thin Solid Films 2011, 519, 4412-4414.

35. Karaman, M.; Çabuk, N. Initiated chemical vapor deposition of $\mathrm{pH}$ responsive poly(2-diisopropylamino)ethyl methacrylate thin films. Thin Solid Films 2012, 520(21), 6484-6488.

36. Bose, R. K.; Lau, K. K. S. Initiated chemical vapor deposition of poly(2-hydroxyethyl methacrylate) hydrogels. Thin Solid Films 2011, 519(14), 4415-4417.

37. Bose, R. K.; Lau, K. K. S. Mechanical properties of ultrahigh molecular weight PHEMA hydrogels synthesized using initiated chemical vapor deposition. Biomacromolecules $\mathbf{2 0 1 0}$, 11, 2126-2122.

38. Ma, M.; Mao, Y.; Gupta, M.; Gleason, K. K.; Rutledge, G. C. Superhydrophobic fabrics produced by electrospinning and chemical vapor deposition. Macromolecules 2005, 38, 9742-9748.

39. Parker, T. C.; Baechle, D.; Demaree, J. D. Polymeric barrier coatings via initiated chemical vapor deposition. Surface \& Coatings Technology 2011, 206(7), 1680-1683.

40. Laird, E. D.; Bose, R. K.; Wang, W.; Lau, K. K. S.; Li, C. Y. Carbon nanotube-directed polytetrafluoroethylene crystal growth via initiated chemical vapor deposition. Macromolecular Rapid Communications 2012, DOI: 10.1002/ marc.201200678. 
41. Riche, C. T.; Marin, B. C.; Malmstadt, N.; Gupta, M. Vapor deposition of cross-linked fluoropolymer barrier coatings onto pre-assembled microfluidic devices. Lab on a Chip 2011, 11(18), 3049-3052.

42. Sreenivasan, R.; Bassett, E. K.; Hoganson, D. M.; Vacanti, J. P.; Gleason, K. K. Ultra-thin gas permeable free-standing and composite membranes for microfluidic lung assist devices. Biomaterials 2011, 32, 3883-3889.

43. Bong, K. W.; Xu, J. J.; Kim, J. H.; Chapin, S. C.; Strano, M. S.; Gleason, K. K.; Doyle, P. S. Non-polydimethylsiloxane devices for oxygen-free flow lithography. Nature Communications 2012, 3, 805.

44. Haller, P. D.; Flowers, C. A.; Gupta, M. Three-dimensional patterning of porous materials using vapor phase polymerization. Soft Matter 2011, 7(6), 2428-2432.

45.Spee, D. A.; Bakker, R.; van der Werf, C. H. M.; van Steenbergen, M. J.; Rath, J. K.; Schropp, R. E. I. Polymer layers by initiated chemical vapor deposition for thin film gas barrier encapsulation. Thin Solid Films 2011, 519(14), 4479-4482.

46. Spee, D.; van der Werf, K.; Rath, J.; Schropp, R. Excellent organic/inorganic transparent thin film moisture barrier entirely made by hot wire CVD at 100 degrees C. Phys Status Solidi-R 2012, 6(4), 151-153.

47. Aresta, G.; Palmans, J.; van de Sanden, M. C. M.; Creatore, M. Evidence of the filling of nano-porosity in SiO2like layers by an initiated-CVD monomer. Microporous and Mesoporous Materials 2012, 151, 434-439.

48. Coclite, A. M.; Gleason, K. K. Initiated PECVD of organosilicon coatings: a new strategy to enhance monomer structure retention. Plasma Processes and Polymers 2012, 9(4), 425-434.

49. Faguet, J.; Lee, E.; Liu, J. J.; Brcka, J.; Akiyama, O. Novel dielectric deposition technology for advanced interconnect with air gap. Proceedings of the 2009 IEEE International Interconnect Technology Conference, 2009, 35-37.

50. Lee, E.; Faguet, J.; Brcka, J.; Akiyama, O.; Liu, J. J.; Toma, D. Single-chamber filament-assisted chemical vapor deposition of polymer and organosilicate films for air gap interconnect formation. Thin Solid Films 2011, 519(14), 4571-4573.

51. Nejati, S.; Lau, K. K. S. Integration of polymer electrolytes in dye sensitized solar cells by initiated chemical vapor deposition. Thin Solid Films 2011, 519(14), 4551-4554.

52. Bakker, R.; Weijers, P.; van der Werf, C. H. M.; Rath, J. K.; Schropp, R. E. I. Doping of oligo(phenyl acetylene) with iodine vapour. Physica Status Solidi a-Applications and Materials Science 2010, 207(3), 647-650.

53. Yoshida, M.; Lahann, J. Smart nanomaterials. ACS Nano 2008, 2(6), 1101-1107.

54. Tekin, H.; Ozaydin-Ince, G.; Tsinman, T.; Gleason, K. K.; Langer, R.; Khademhosseini, A.; Demirel, M. C. Responsive microgrooves for the formation of harvestable tissue constructs. Langmuir 2011, 27, 5671-5679.

55. Petruczok, C. D.; Choi, H. J.; Yang, S. Y.; Asatekin, A.; Gleason, K. K.; Barbastathis, G. Fabrication of a microscale device for detection of nitroaromatic compounds. Journal of Microelectromechanical Systems 2012, PP(99), 1-8.

56. Ozaydin-Ince, G.; Dubach, J. M.; Gleason, K. K.; Clark, H. A. Microworm optode sensors limit particle diffusion to enable in vivo measurements. PNAS 2011, 108, 2656-2661.

57. Montero, L.; Gabriel, G.; Guimerà, A.; Villa, R.; Gleason, K. K.; Borrós, S. Increasing biosensor response through hydrogel thin film deposition: Influence of hydrogel thickness. Vacuum 2012, 86(12), 2102-2104.

58. Yagüe, J. L.; Gleason, K. K. Systematic control of mesh size in hydrogels by initiated chemical vapor deposition. Soft Matter 2012, 8(10), 2890-2894.

59. Bose, R. K.; Heming, A. M.; Lau, K. K. S. Microencapsulation of a crop protection compound by initiated chemical vapor deposition. Macromolecular Rapid Communications 2012, 33(17), 1375-1380.

60. Kwong, P.; Flowers, C. A.; Gupta, M. Directed deposition of functional polymers onto porous substrates using metal salt inhibitors. Langmuir 2011, 27(17), 10634-10641.

61. Yin, J.; Yagüe, J. L.; Eggenspieler, D.; Gleason, K. K.; Boyce, M.C. Deterministic order in surface micro-topologies through sequential wrinkling. Advanced Materials 2012, 24 (40), 5441-5446.

62. Chen, B.; Seidel, S.; Hori, H.; Gupta, M. Self-assembly of pillars modified with vapor deposited polymer coatings. ACS Applied Materials \& Interfaces 2011, 3(11), 4201-4205.

63. Petruczok, C. D.; Gleason, K. K. Initiated chemical vapor deposition-based method for patterning polymer and metal microstructures on curved substrates. Advanced Materials 2012, 24, 6445-6450.

\section{WHAT DO YOU THINK?}

To discuss this paper, please email up to 500 words to the managing editor at sufi@icepublishing.com

Your contribution will be forwarded to the author(s) for a reply and, if considered appropriate by the editor-inchief, will be published as a discussion in a future issue of the journal.

ICE Science journals rely entirely on contributions sent in by professionals, academics and students coming from the field of materials science and engineering. Articles should be within 5000-7000 words long (short communications and opinion articles should be within 2000 words long), with adequate illustrations and references. To access our author guidelines and how to submit your paper, please refer to the journal website at www.icevirtuallibrary.com/sufi 